PROCEEDINGS OF THE

AMERICAN MATHEMATICAL SOCIETY

Volume 136, Number 11, November 2008, Pages 3953-3956

S 0002-9939(08)09377-5

Article electronically published on June 2, 2008

\title{
UPPER BOUND FOR ISOMETRIC EMBEDDINGS $\ell_{2}^{m} \rightarrow \ell_{p}^{n}$
}

\author{
YU. I. LYUBICH
}

(Communicated by N. Tomczak-Jaegermann)

\begin{abstract}
The isometric embeddings $\ell_{2 ; \mathbb{K}}^{m} \rightarrow \ell_{p ; \mathbb{K}}^{n}(m \geq 2, p \in 2 \mathbb{N})$ over a field $\mathbb{K} \in\{\mathbb{R}, \mathbb{C}, \mathbb{H}\}$ are considered, and an upper bound for the minimal $n$ is proved. In the commutative case $(\mathbb{K} \neq \mathbb{H})$ the bound was obtained by Delbaen, Jarchow and Pełczyński (1998) in a different way.
\end{abstract}

Let $\mathbb{K}$ be one of three fields $\mathbb{R}, \mathbb{C}, \mathbb{H}$ (real, complex or quaternion). Let $\mathbb{K}^{n}$ be the $\mathbb{K}$-linear space consisting of columns $x=\left[\xi_{i}\right]_{1}^{n}, \xi_{i} \in \mathbb{K}$, with the right (for definiteness) multiplication by scalars $\alpha \in \mathbb{K}$. The normed space $\ell_{p ; \mathbb{K}}^{n}$ is $\mathbb{K}^{n}$ provided with the norm

$$
\|x\|_{p}=\left(\sum_{k=1}^{n}\left|\xi_{i}\right|^{p}\right)^{1 / p}, \quad 1 \leq p<\infty .
$$

For $p=2$ this space is Euclidean, $\|x\|_{2}=\sqrt{\langle x, x\rangle}$, where the inner product $\langle x, y\rangle$ of $x$ and a vector $y=\left[\eta_{i}\right]_{1}^{n}$ is

$$
\langle x, y\rangle=\sum_{i=1}^{n} \overline{\xi_{i}} \eta_{i} .
$$

An isometric embedding $\ell_{2 ; \mathbb{K}}^{m} \rightarrow \ell_{p ; \mathbb{K}}^{n}, 2 \leq m \leq n$, may exist only if $p \in 2 \mathbb{N}=$ $2,4,6, \ldots$; see 3 for $\mathbb{K}=\mathbb{R}$ and 4 for any $\mathbb{K}$. Conversely, under these conditions for $m$ and $p$, there exists an $n$ such that $\ell_{2 ; \mathbb{K}}^{m}$ can be isometrically embedded into $\ell_{p ; \mathbb{K}}^{n}$; see [6] (and also [5, 7]) for $\mathbb{K}=\mathbb{R},[2]$ for $\mathbb{K}=\mathbb{C}$, and [4] for $\mathbb{K}=\mathbb{H}, \mathbb{C}$ and $\mathbb{R}$ simultaneously. The proofs of existence in these papers also yield some upper bounds for the minimal $n=N_{\mathbb{K}}(m, p)$. According to [4, these bounds can be joined in the inequality

$$
N_{\mathbb{K}}(m, p) \leq \operatorname{dim} \Phi_{\mathbb{K}}(m, p),
$$

where $\Phi_{\mathbb{K}}(m, p)$ is the space of homogeneous polynomials (forms) $\phi(x)$ over $\mathbb{R}$ of degree $p$ in real coordinates on $\mathbb{K}^{m}$ such that $\phi(x \alpha)=\phi(x)$ for all $\alpha \in \mathbb{K},|\alpha|=$ 1. For $\mathbb{K}=\mathbb{R}$ the latter condition is fulfilled automatically since $p \in 2 \mathbb{N}$, so $\Phi_{\mathbb{R}}(m, p)$ consists of all forms of degree $p$ on $\mathbb{R}^{m}$. The space $\Phi_{\mathbb{C}}(m, p)$ coincides with that which was used in [2]. Note that in all cases $\operatorname{dim} \Phi_{\mathbb{K}}(m, p)$ can be explicitly expressed through binomial coefficients. (All the formulas are brought together in [4, Theorem 2].)

Received by the editors August 1, 2007, and, in revised form, October 3, 2007.

2000 Mathematics Subject Classification. Primary 46B04.

Key words and phrases. Isometric embeddings, quaternion spaces.

(C)2008 American Mathematical Society Reverts to public domain 28 years from publication 
In the present paper we prove that

$$
N_{\mathbb{K}}(m, p) \leq \operatorname{dim} \Phi_{\mathbb{K}}(m, p)-1 .
$$

For $\mathbb{K}=\mathbb{R}$ and $\mathbb{C}$ this result (in terms of binomial coefficients) was obtained by Delbaen, Jarchow and Pełczyński [1] as a by-product of the proof of their Theorem B. Their rather complicated technique essentially uses the commutativity of the field $\mathbb{K}$, so it is not applicable to $\mathbb{K}=\mathbb{H}$. Our proof of (2) is general and elementary. Let us start with two lemmas, the first of which is well known.

Lemma 1. A linear mapping $f: \ell_{2 ; \mathbb{K}}^{m} \rightarrow \ell_{p ; \mathbb{K}}^{n}$ is isometric if and only if there is a system of vectors $u_{k} \in \ell_{2 ; \mathbb{K}}^{m}, 1 \leq k \leq n$, such that the identity

$$
\sum_{k=1}^{n}\left|\left\langle u_{k}, x\right\rangle\right|^{p}=\langle x, x\rangle^{p / 2}
$$

holds for $x \in \ell_{2 ; \mathbb{K}}^{m}$.

Proof. A general form of $f$ as a linear mapping is $f x=\left[\left\langle u_{k}, x\right\rangle\right]_{1}^{n}$, where $\left(u_{k}\right)_{1}^{n}$ is a system of vectors from $\ell_{2 ; \mathbb{K}}^{m}$ (called the frame of $f[4,5]$ ). The identity (3) is nothing but $\|f x\|_{p}=\|x\|_{2}$.

An isometric embedding $\ell_{2 ; \mathbb{K}}^{m} \rightarrow \ell_{p ; \mathbb{K}}^{n}$ is called minimal if $n=N_{\mathbb{K}}(m, p)$.

Lemma 2. If $f$ is minimal and $\left(u_{k}\right)_{1}^{n}$ is its frame, then the functions $\left|\left\langle u_{k}, x\right\rangle\right|^{p}$ are linearly independent.

Proof. Let

$$
\sum_{k=1}^{n} \omega_{k}\left|\left\langle u_{k}, x\right\rangle\right|^{p}=0
$$

with some real $\omega_{k}, \max \omega_{k}=1$, and let $\omega_{n}=1$ for definiteness. By subtraction of (44) from (3) we get

$$
\sum_{k=1}^{n-1}\left(1-\omega_{k}\right)\left|\left\langle u_{k}, x\right\rangle\right|^{p}=\langle x, x\rangle^{p / 2}
$$

i.e.

$$
\sum_{k=1}^{n-1}\left|\left\langle v_{k}, x\right\rangle\right|^{p}=\langle x, x\rangle^{p / 2},
$$

where $v_{k}=u_{k}\left(1-\omega_{k}\right)^{1 / p}$. This contradicts the minimality of $f$.

Remark 3. Since all functions $|\langle\cdot, x\rangle|^{p}$ belong to $\Phi_{\mathbb{K}}(m, p)$, the inequality (1) immediately follows from Lemma 2. However, the existence of an isometric embedding $\ell_{2 ; \mathbb{K}}^{m} \rightarrow \ell_{p ; \mathbb{K}}^{n}$ is assumed in this context.

Now we proceed to the proof of (2) .

Proof. Let $f: \ell_{2 ; \mathbb{K}}^{m} \rightarrow \ell_{p ; \mathbb{K}}^{n}$ be a minimal isometric embedding. Then, according to (11), $n \leq \operatorname{dim} \Phi_{\mathbb{K}}(m, p)$. We have to prove that the equality is impossible.

Suppose the contrary. Then the system $\left(\left|\left\langle u_{k}, x\right\rangle\right|^{p}\right)_{1}^{n}$ corresponding to the frame of $f$ is a basis of $\Phi_{\mathbb{K}}(m, p)$ by Lemma 2 In particular, there is an expansion

$$
\left(\sum_{i=1}^{m} \lambda_{i}\left|\xi_{i}\right|^{2}\right)^{p / 2}=\sum_{k=1}^{n} a_{k}\left(\lambda_{1}, \ldots, \lambda_{m}\right)\left|\left\langle u_{k}, x\right\rangle\right|^{p},
$$


where $\left(\lambda_{i}\right)_{1}^{m} \in \mathbb{R}^{m}$ and $a_{k}$ are some functions of these parameters.

Now we introduce the inner product

$$
\left(\phi_{1}, \phi_{2}\right)=\int_{S} \phi_{1}(x) \phi_{2}(x) d \sigma(x) \quad\left(\phi_{1}, \phi_{2} \in \Phi_{\mathbb{K}}(m, p)\right),
$$

where $\sigma$ is the standard measure on the unit sphere $S \subset \ell_{2 ; \mathbb{K}}^{m}$. In the Euclidean space $\Phi_{\mathbb{K}}(m, p)$ we have the basis $\left(\theta_{k}(x)\right)_{1}^{n}$ dual to $\left(\left|\left\langle u_{k}, x\right\rangle\right|^{p}\right)_{1}^{n}$. This allows us to represent the coefficients $a_{k}$ as

$$
a_{k}\left(\lambda_{1}, \ldots, \lambda_{m}\right)=\int_{S}\left(\sum_{i=1}^{m} \lambda_{i}\left|\xi_{i}\right|^{2}\right)^{p / 2} \theta_{k}(x) d \sigma(x) .
$$

Hence, $a_{k}\left(\lambda_{1}, \ldots, \lambda_{m}\right)$ are forms of degree $p / 2$; a fortiori, they are continuous.

Denote by $\mathbb{R}_{+}^{m}$ the open coordinate cone in $\mathbb{R}^{m}$, so $\mathbb{R}_{+}^{m}=\left\{\left(\lambda_{i}\right)_{1}^{m} \subset \mathbb{R}^{m}: \lambda_{1}>\right.$ $\left.0, \ldots, \lambda_{m}>0\right\}$. We prove that on $\mathbb{R}_{+}^{m}$ all $a_{k}\left(\lambda_{1}, \ldots, \lambda_{m}\right)>0$ or equivalently, $\hat{a}\left(\lambda_{1}, \ldots, \lambda_{m}\right) \equiv \min _{k} a_{k}\left(\lambda_{1}, \ldots, \lambda_{m}\right)>0$. Suppose the contrary: let $\hat{a}\left(\gamma_{1}, \ldots, \gamma_{m}\right) \leq$ 0 for some $\left(\gamma_{i}\right)_{1}^{m} \subset \mathbb{R}_{+}^{m}$. On the other hand, $\hat{a}(1, \ldots, 1)=1$ by comparing (3) to (5) with all $\lambda_{i}=1$. Since $\hat{a}$ is continuous, we have $\hat{a}\left(\mu_{1}, \ldots, \mu_{m}\right)=0$ for some $\left(\mu_{i}\right)_{1}^{m} \subset \mathbb{R}_{+}^{m}$. But the latter means that all $a_{k}\left(\mu_{1}, \ldots, \mu_{m}\right) \geq 0$ and at least one of them is zero, say $a_{n}\left(\mu_{1}, \ldots, \mu_{m}\right)=0$. Therefore,

$$
\left(\sum_{i=1}^{m} \mu_{i}\left|\xi_{i}\right|^{2}\right)^{p / 2}=\sum_{k=1}^{n-1} a_{k}\left(\mu_{1}, \ldots, \mu_{m}\right)\left|\left\langle u_{k}, x\right\rangle\right|^{p},
$$

whence

$$
\langle z, z\rangle^{p / 2}=\sum_{k=1}^{n-1}\left|\left\langle v_{k}, z\right\rangle\right|^{p}
$$

where

$$
z=\mathcal{D} x, \quad v_{k}=\left(a_{k}\left(\mu_{1}, \ldots, \mu_{m}\right)\right)^{1 / p} \mathcal{D}^{-1} u_{k}
$$

and $\mathcal{D}$ is the diagonal matrix with entries $\mu_{1}^{1 / 2}, \ldots, \mu_{m}^{1 / 2}$. By Lemma 1 the identity (16) means that the system $\left(v_{k}\right)_{1}^{n-1}$ is the frame of an isometric embedding $\ell_{2 ; \mathbb{K}}^{m} \rightarrow$ $\ell_{p ; \mathbb{K}}^{n-1}$. This contradicts the minimality of $n$. As a result, all $a_{k}\left(\lambda_{1}, \ldots, \lambda_{m}\right) \geq 0$ for $\lambda_{1} \geq 0, \ldots, \lambda_{m} \geq 0$, i.e. on the closed coordinate cone.

Now we take $\xi_{1}=1$ and $\xi_{i}=0$ for all $i \geq 2$, so $x=e_{1}$, the first vector from the canonical basis of $\ell_{2 ; \mathbb{K}}^{m}$. In this setting (15) reduces to

$$
\lambda_{1}^{p / 2}=\sum_{k=1}^{n} a_{k}\left(\lambda_{1}, \ldots, \lambda_{m}\right)\left|\left\langle u_{k}, e_{1}\right\rangle\right|^{p} .
$$

(Recall that $m \geq 2$.) This yields

$$
\sum_{k=1}^{n} a_{k}\left(0, \lambda_{2}, \ldots, \lambda_{m}\right)\left|\left\langle u_{k}, e_{1}\right\rangle\right|^{p}=0 .
$$

Assume all $\left\langle u_{k}, e_{1}\right\rangle \neq 0$. Since for $\lambda_{2}>0, \ldots, \lambda_{m}>0$ all $a_{k}\left(0, \lambda_{2}, \ldots, \lambda_{m}\right) \geq 0$, all of them are equal to zero. Hence, the right side of the identity (5) vanishes as long as $\lambda_{1}=0$, in contrast to the function on the left side, a contradiction. To finish the proof we only have to show that the assumption $\left\langle u_{k}, e_{1}\right\rangle \neq 0,1 \leq k \leq n$, is not essential. 
First, note that all $u_{k} \neq 0$; otherwise, the number $n$ in (3) would be reduced. Therefore, the sets $\left\{x:\left\langle u_{k}, x\right\rangle=0\right\}, 1 \leq k \leq n$, are hyperplanes in $\ell_{2 ; \mathbb{K}}^{m}$. Their union is different from the whole space. Hence, there is a vector $e$ such that all $\left\langle u_{k}, e\right\rangle \neq 0,\|e\|_{2}=1$. This $e$ can be represented as $e=g e_{1}$ where $g$ is an isometry of the space $\ell_{2 ; \mathbb{K}}^{m}$. Indeed, this space is Euclidean, so its isometry group is transitive on the unit sphere. Thus, all $\left\langle g^{-1} u_{k}, e_{1}\right\rangle \neq 0$. On the other hand, $\left(g^{-1} u_{k}\right)_{1}^{n}$ is the frame of the isometric embedding $f g: \ell_{2 ; \mathbb{K}}^{m} \rightarrow \ell_{p ; \mathbb{K}}^{n}$.

\section{REFERENCES}

1. F. Delbaen, H. Jarchow, and A. Pełczyński, Subspaces of $L_{p}$ isometric to subspaces of $l_{p}$, Positivity 2 (1998), no. 4, 339-367. MR:1656109 (99m:46071)

2. H. König, Isometric imbeddings of Euclidean spaces into finite-dimensional $l_{p}$-spaces, Banach Center Publ., vol. 34, Polish Acad. Sci., Warsaw, 1995, pp. 79-87.

3. Yu. I. Lyubich, The boundary spectrum of contractions in Minkowski spaces, Sibirsk. Mat. Ž. 11 (1970), 358-369; English Transl., Siberian Math. J. 11 (1970), no. 2, 271-279. MR0270191 $(42: 5083)$

4. Yu. I. Lyubich and O. A. Shatalova, Isometric embeddings of finite-dimensional $l_{p}$-spaces over the quaternions, St. Petersburg Math. J. 16 (2005), no. 1, 9-24. MR.2068351 (2005d:46018)

5. Yu. I. Lyubich and L. N. Vaserstein, Isometric embeddings between classical Banach spaces, cubature formulas, and spherical designs, Geom. Dedicata 47 (1993), no. 3, 327-362. MR.1235223 (94j:46017)

6. V. D. Milman, A few observations on the connections between local theory and some other fields, Geometric aspects of functional analysis (1986/87), Lecture Notes in Math., vol. 1317, Springer, Berlin, 1988, pp. 283-289. MR.950988 (89h:52004)

7. B. Reznick, Sums of even powers of real linear forms, Mem. Amer. Math. Soc. 96 (1992), no. 463. MR1096187 (93h:11043)

Department of Mathematics, Technion, 32000, Haifa, Israel

E-mail address: lyubich@tx.technion.ac.il 